\title{
Non-invasive evaluation of obstruction after ureteroscopic stone removal: Role of renal resistive index assessment
}

\author{
Bilal Eryildirim ${ }^{1}$, Ahmet Sahan ${ }^{1}$, Özlem Türkoğlu ${ }^{2}$, Murat Tuncer ${ }^{1}$, Övünç Kavukoğlu ${ }^{1}$, \\ Berkan Simsek $^{1}$, Alkan Cubuk ${ }^{1}$, Kemal Sarıca ${ }^{3}$ \\ ${ }^{1}$ Health Sciences University, Kartal Dr. Lutfi Kirdar Training and Research Hospital, Urology Clinic, Istanbul, Turkey; \\ ${ }^{2}$ Health Sciences University, Sultan Abdulhamid Han Training and Research Hospital, Radiology Clinic, Istanbul, Turkey; \\ ${ }^{3}$ Biruni University, Medical School, Urology Clinic, Istanbul, Turkey.
}

\begin{abstract}
Summary Objectives: The aim of this study is to evaluate prediction of postoperative ureteral obstruction needing ureteral stent insertion by evaluating the resistive index (RI) values and the grade of hydronephrosis. Material and Methods: A total of 66 adult patients undergoing stentless endoscopic ureteral stone treatment (URS) between January 2018 and January 2019 were included in this prospective study. Preoperative patient and stone characteristics were noted. All patients were evaluated with renal Doppler ultrasonography study to assess degree of hydronephrosis and RI values. A renal Doppler ultrasonography was repeated at postoperative $1^{\text {st }}, 3^{\text {rd }}$ and $7^{\text {th }}$ days. Changes in both RI and hydronephrosis levels before and after the procedures were noted. On the postoperative 7th day, patients were divided into two groups including obstructive and non-obstructive cases according to RI values assessed where a RI value of 0.7 was accepted as the cut-off for obstruction. The preoperative and perioperative characteristics of both groups were evaluated in a comparative manner.

Results: The mean patient age was $43.6 \pm 1.72$ years. Significant improvements were noted in RI and grade of hydronephrosis after the operation. The grade of hydronephrosis and RI values were found to improve more significantly on postoperative $3^{\text {rd }}$ day when compared to the postoperative $7^{\text {th }}$ day $(p<0.01$ and $p<0.01)$. A significant correlation was detected between the grade of hydronephrosis (>grade 2) and obstructive RI values $(>0.7)$ in each postoperative visits (p: 0.001). RI values (>0.7) at postoperative seventh days were correlated with larger mean stone size, increased ureteral wall thickness, increased diameter of the ureter proximal to the stone, and longer duration of the operation. Preoperative high-grade hydronephrosis indicated obstructive RI values at postoperative seventh day $(p=0.001)$ Conclusion: Changes in $R I$ values on Doppler sonography and the grade of hydronephrosis may be a guiding parameter in assessing postoperative ureteral obstruction.
\end{abstract}

KEY WORDS: Ureteral obstruction; Resistive index; Renal Doppler ultrasonography.

Submitted 2 March 2020; Accepted 15 March 2020

\section{INTRODUCTION}

Urolithiasis is a common disease that affects $5-10 \%$ of the general population (1). Of all the stones diagnosed ureteral calculi sizing larger than $5 \mathrm{~mm}$ may cause obstruction and colic pain requiring immediate management. Early intervention on this aspect, not only protects renal morphological and functional integrity but also enables the patient to return to normal life quickly $(2,3)$. Ureteroscopic procedures have an important place not only in the diagnosis but especially in the treatment of ureteral stones. Although the procedure is accepted as a minimally invasive approach; some certain complications and procedure-related complaints could be noted in a certain percent of the cases. Persistence of the obstruction after ureteroscopic procedures secondary to the edema formation, presence of coagulum, and residual stone fragments is a major problem after this modality. Follow-up of the ureteral obstruction in a non-invasive manner constitutes a dilemma both during preoperative and also postoperative periods.

Renal Doppler ultrasonography (USG) may be one of the non-invasive diagnostic methods to help us in evaluating the degree as well as the course of the ureteral obstruction following endoscopic ureteral stone treatment. It has been well shown that changes in the degree of hydronephrosis and resistive index values $(\Delta \%)$ after ureteroscopic stone removal could provide important information regarding the severity (grade) of obstruction $(4,5)$.

Insertion of an ureteral stent after ureteroscopic procedures can be a rational option to avoid postoperative obstruction. However, accumulated data so far have clearly shown that despite their protective effects, ureteral stents may certainly have some distressing problems which have been classified as stent-related symptoms $(6,7)$. Due to this fact, the insertion of a stent after these procedures is optional depending on the preference of the surgeon. On the other hand, again, there is no commonly accepted consensus with established certain criteria for the necessity of a ureteral stent after ureteroscopic procedures $(8,9)$. Studies have demonstrated that not all of the dilatation detected in the upper urinary tract after ureteral stone removal will reflect a true obstruction and residual dilation is a commonly observed scenario. Taking this fact into account again no reliable criteria with certain parameters have been reported to predict which patients will require ureteral DJ stent placement due to ureteral obstruction during follow-up after endoscopic ureteral stone surgery.

To fill this gap, in this present prospective study, we aimed to investigate the predict the presence of postoperative ureteral obstruction after ureteral stone removal by evaluating the resistive index values and the grade of hydronephrosis. 


\section{Patients AND methods}

A total of 66 adult patients undergoing stentless endoscopic ureteral stone treatment for ureteral stones between January 2018 and January 2019 were included in the study. Patients with previous stone surgery including stent placement and auxiliary procedures, congenital anomalies, active urinary tract infection, pregnancy, renovascular disease, or renal insufficiency were all excluded from the program.

Approval for the study protocol was obtained from our Local Hospital Ethics Committee and all cases were well informed about the procedure from all aspects in detail and informed consent was obtained before the intervention (Local ethical approval no: 2017/514/115/2).

In addition to a detailed medical history; a careful physical examination was done and biochemical examinations including renal functional tests, urine analysis together with urine culture sensitivity tests were performed. Although a non-contrast computed tomography (NCCT) was performed in all cases during a colic attack; sonography, plain X-ray of the kidney, ureter, and bladder (KUB) and excretory urography were done when necessary.

In addition to these evaluations, renal color doppler sonography was done preoperatively in all cases in the supine position using $3.5-5 \mathrm{MHz}$ transducer of MINDRAY ${ }^{\circledR}$ Realtime ultrasound machine Model DC-6 (ShenzhenMindrayBiomedical electronics, Nanshan, Shenzhen, China). The presence of hydronephrosis was evaluated in each case on Bmode and graded as described by Piazzese et al. (10). Thereafter, the Doppler study (color and spectral) of the interlobar arteries was done to calculate the renal resistive index value as [(peak systolic velocity \pm end-diastolic velocity)/peak systolic velocity] $(11,12)$.

Preoperative stone and patient-related parameters such as stone size and localization, the diameter of the ureter proximal to the stone, the ureteral wall thickness at the site of the ureteral stone (UWT), the grade of hydronephrosis and RI values were all assessed and recorded (3).

All ureteroscopic stone removal procedures were performed under general anesthesia by using a semirigid $8 \mathrm{Fr}$ ureteroscope (Karl Storz, Tuttlingen, Germany). All cases received 1 gr of Cephazolin injection for prophylaxis before the procedure. Stone fragmentation was accomplished by using the holmium-YAG laser. Perioperative data including duration of operation, stone-free rate, and hospitalization time were recorded. The interval between initial colic pain and surgery was also recorded.

All patients were scheduled for a postoperative follow-up evaluation after $1^{\text {st }}$ day, $3^{\text {rd }}$ day and $7^{\text {th }}$ day following the procedures. A Doppler USG was performed at each visit to assess the grade hydronephrosis and RI values. Ultrasonography was carried out by the same senior radiologist. Changes in both RI and hydronephrosis levels before and after the procedures were given as "percentage of change" $(\Delta \%)$ (Difference between a timely measurement with basal level/basal level x100) (10).

On the postoperative $7^{\text {th }}$ day, patients were divided into two groups including obstructive and non-obstructive cases according to RI values assessed where a RI value of 0.7 was accepted as the cut-off for obstruction (10). The preoperative and perioperative characteristics of both groups were evaluated in a comparative manner.

\section{Statistical analysis}

The IBM SPSS version 20.0 (IBM Co., Armonk, NY) was used for statistical analysis. Chi-square test was applied to evaluate categorical data and the two-sided P-value was used in inference, and $p<0.05$ was accepted as significant. The variables were investigated using visual (histogram) and analytic methods (Kolmogorov-Smirnow) to determine normal distribution. Descriptive analyses were presented using means and standard deviation.

The ANOVA was used to compare normally distributed parameters.

\section{RESULTS}

Of all the 66 patients ( 40 men and 26 women) evaluated 36 cases had a stone on the right and 30 cases on the left side. The overall mean patient age was $43.6 \pm 1.72$ years with a mean BMI value of $26.3 \pm 0.4 \mathrm{~kg} / \mathrm{m}^{2}$. Changes in both the RI values and the grade of hydronephrosis before and after the ureterolithotripsy were significantly improved. The grade of hydronephrosis and RI values were found to improve more significantly on postoperative 3rd day when compared to the postoperative $7^{\text {th }}$ day (Figures 1, 2). A comparison of hydronephrosis grade and resistive index values in conjunction with the timing of the follow-up period is being shown in Table 1.

A hydronephrosis grade of 2 and more were detected in 46 of the patients preoperatively. While 30 of these patients were diagnosed as on day 1, 18 patients were on postoperative day 3 and 10 patients on postoperative day 7. A significant correlation was detected between the grade of hydronephrosis (> grade 2 ) and obstructive RI values (>0.7) (p: 0.001).

According to the resistive index value detected, 42 patients were found to have an obstructive pattern at preoperatively and 26 patients were found to have an obstructive pattern at postoperative day 7 . Further evaluation of our findings clearly demonstrated that obstructive RI values $>0.7$ ) at postoperative 7 days were well

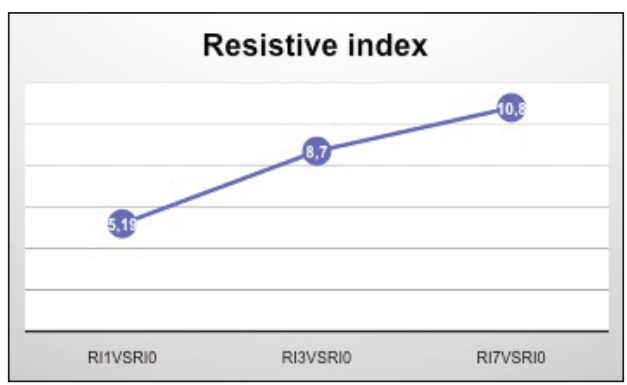

Figure 1.
Delta
resistive
index
changes are
shown below
during
the follow-up.

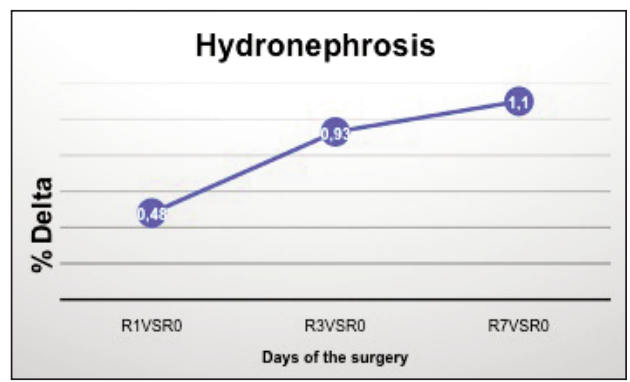

Figure 2 Delta hydronephrosis grade changes are shown below during the follow-up. 
Table 1.

Comparison of hydronephrosis and resistive index values among the days of the surgery.

\begin{tabular}{|c|c|c|c|c|c|c|c|c|}
\hline \multicolumn{3}{|c|}{ Grade of hydronephrosis } & \multicolumn{6}{|c|}{ Resistive index values } \\
\hline & \multicolumn{2}{|r|}{ RI 0} & \multicolumn{2}{|r|}{ RI 1} & \multicolumn{2}{|r|}{ RI 3} & \multicolumn{2}{|r|}{ RI 7} \\
\hline 0 & 2 & 0.680 & 12 & 0.611 & 28 & 0.583 & 30 & 0.562 \\
\hline 1 & 18 & 0.664 & 24 & 0.662 & 20 & $0.680^{*}$ & 26 & $0.695^{*}$ \\
\hline 2 & 38 & $0.729^{\&}$ & 28 & $0.725^{*}, \&$ & 18 & $0.757^{*}, \&$ & 10 & $0.766^{*}, \&$ \\
\hline 3 & 8 & $0.840^{*}, \& .+$ & 2 & $0.860^{*}, \&$ & 0 & - & 0 & - \\
\hline Total & 66 & 0.723 & 66 & 0.686 & 66 & 0.660 & 66 & 0.645 \\
\hline pvalue & & 0.01 & & 0.01 & & 0.01 & & 0.01 \\
\hline
\end{tabular}

Table 2.

Comparison of obstructive resistive index value based on $R I 7^{\text {th }}$ day value to pre-operative parameters.

\begin{tabular}{|c|c|c|c|c|}
\hline & & $\mathrm{RI}<0.7(\mathrm{n}: 40)$ & $\mathrm{RI}>0.7(\mathrm{n}: 26)$ & $\mathrm{p}$ value \\
\hline Ureteral stone diameter & & $6.8(1.6)$ & $8.5(1.4)$ & 0.01 \\
\hline Ureteral wall thickness (UWT) & & $3.3(1.2)$ & $4.9(0.6)$ & 0.01 \\
\hline Diameter of theureter & & $9.2(3.2)$ & $13.6(3.06)$ & 0.01 \\
\hline Duration of theoperation & & $36.2(7.1)$ & $48.0(5.1)$ & 0.01 \\
\hline Stone localization (upper/lower) & & $10 / 30$ & $10 / 16$ & 0.245 \\
\hline Preoperative grade & 0 & 2 & 0 & \\
\hline \multirow[t]{3}{*}{ of hydronephrosis } & 1 & 14 & 0 & 0.001 \\
\hline & 2 & 24 & 8 & \\
\hline & 3 & 0 & 18 & \\
\hline
\end{tabular}

correlated with larger mean stone size, increased UWT, increased diameter of the ureter proximal to the stone, and longer duration of the operation (Table 2).

Additionally, the presence of preoperative high-grade hydronephrosis seemed to increase the likelihood of postoperative obstruction according to resistive index values assessed at postoperative days $7(p=0.001)$.

\section{Discussion}

Endourological procedures were introduced to the clinical practice in the 1980s and since then they have been used as widely accepted and reliable methods for ureteral stone treatment with limited complications (14). As a result of the technological improvements, ureterorenoscopes became thinner, and advanced visualization quality resulted in increased success and decreased complication rates. Despite a successful ureteroscopic procedure, however, published data have demonstrated that ipsilateral colic pain can be observed due to edema and coagulum formation, residual stone fragments which may cause a temporary urinary obstruction and lead to the symptoms similar to the preoperative period in these patients. In light of these facts, it will be very useful to predict the possibility of postoperative obstruction during the preoperative evaluation period and plan to insert a ureteral stent after successful stone removal. On the other hand, evaluation and follow-up of possible obstructive status after such interventions with minimal or non-invasive means carry considerable importance for the involved cases. Renal Doppler USG and RI value assessment can be a useful tool in the non-invasive evaluation of upper urinary tract obstruction on this aspect $(4,15)$. Related with this issue,
Apoku et al reported that renal Doppler sonography is highly sensitive and specific for the diagnosis of obstructive uropathy. Increased resistive index of the obstructed kidney may be a useful diagnostic tool in situations where intravenous urography cannot be done or is contraindicated. Their findings indicated a sensitivity and specificity of $86.7 \%$ and $90 \%$ respectively following urinary obstruction, an increase in the pressure of the intrarenal collecting system will occur. The result of increased renovascular resistance will eventually cause a reduction in the renal blood flow. An increase in intra-renal vascular resistance diminishes diastolic blood flow velocity in intrarenal arteries which will subsequently cause an increase in the RI values noted (16). Similarly, Patti et al. have emphasized that $\mathrm{RI}$ is a good index of obstruction in children with unilateral hydronephrosis which has been found to be well correlated with the results of diuretic renography in such cases (12). Thus, all these findings indicate the possible and effective use of RI value assessment in the follow-up of upper tract obstruction.

In our current prospective study, we evaluated the presence and degree of obstruction in the upper urinary system with renal Doppler USG (RI values and grade of hydronephrosis) before and after (1, 3, and 7 days) ureterolithotripsy in cases with ureteric stones. We observed a gradual improvement in RI values and grade of hydronephrosis following the removal of ureteral calculi. The change in RI values and grade of hydronephrosis after stone removal $(\Delta \%)$ was found to be statistically significant. Furthermore, the improvement in RI values after intervention with significant decrease were well correlated with the decreasing grade of hydronephrotic status during the postoperative follow up period. Our current findings implicated that, the RI values and grade of hydronephrosis are convenient and useful parameters in the noninvasive follow-up of obstruction status after ureterolithotripsy.

Routine ureteral stent insertion is not recommended after an uncomplicated URS, as it both increases the cost and lowers the patients' quality of life. According to EUA guidelines, ureteral stents should be inserted in patients with any operative complication (bleeding, perforation, unsuccessful ureterolitotripsy, and ureteral injury) and in all doubtful situations to avoid the possible risk of postoperative complications $(8,9,17,18)$.

However finding the answer to outline these "doubtful cases as well as to avoid stressful emergencies" mentioned in the EAU urolithiasis guideline, we need to define some predictive parameters to assess such situations. It is a known fact that many patients may refer to the emergency department with colic flank pain after a successful endoscopic ureteral stone surgery. Possible causes of partial ureteral obstruction in such cases are edema formation in the ureteral wall or presence of a coagulum in the lumen of the ureter. Preoperative prediction of such factors (if possible) is highly important to decide on the placement of a ureteral stent following stone removal. In the light of the RI values obtained, 26 patients (without ant stent in place) had obstructive patterns at postoperative day 7 in our study. Of these patients classified as "obstructed" according to the RI values; eight of them had grade 2 hydronephrosis and 18 patients had grade 3 hydronephrosis in the preoperative period. 
Based on our current findings, we can say that the grade of preoperative hydronephrosis may be associated with the postoperative obstructive pattern and ureteral stent placement might be more beneficial in these patients. Additionally, patients with obstructive RI values at postoperative 7 days did also have increased mean stone size, UWT values, the diameter of the ureter proximal to the stone during preoperative evaluations along with longer mean operative duration. These data clearly indicate that postoperative restoration of the obstruction may take some more time if the stone burden, UWT value, the diameter of the ureter proximal to the stone is high and the operation time is longer. Our study has certain limitations. First of all the number of cases included in the study program is limited. Also, long-term follow-up data are lacking in the groups. However, taking the lack of publications regarding this issue and the first introduction of such parameters to assess the factors affecting postoperative ureteral obstruction; we believe that our current findings will certainly be contributive enough to the existing literature on this critical subject. Lastly, it would be interesting to compare cases with high RI $>0.7$ and the ones with no or mild upper tract dilatation, to help the urologist to decide to place stent rather than wait for the obstruction to resolve by itself in these cases. However, we were not able to make such a comparison due to the limited number of patients in our groups.

\section{Conclusions}

Based on our current findings we may state that the changes in RI values on Doppler sonography and the grade of hydronephrosis may be a guiding parameter in assessing whether obstruction is removed after endoscopic ureteral stone treatment. If the stone burden is high, operation time is long and the stone is impacted to the ureteric wall, postoperative restoration of obstruction may take longer than anticipated. We believe that in addition to changes in the degree of hydronephrosis outlined, the assessment of renal RI values may provide us further information to differentiate the obstructive pattern after ureteroscopic surgery prior to ureteral catheter placement.

\section{REFERENCES}

1. Teichman JM. Clinical practice. Acute renal colic from ureteral calculus. N Engl J Med. 2004; 350:684-93.

2. Joshi HB, Obadeyi OO, Rao PN. A comparative analysis of nephrostomy, JJ stent and urgent in situ extracorporeal shock wave lithotripsy for obstructing ureteric stones. BJU Int. 1999; 84:264-9.

3. Yencilek F, Sarica K, Erturhan S, et al. Treatment of ureteral calculi with semirigid ureteroscopy: where should we stop? Urol Int. 2010; 84:260-4.

4. Apoku IN, Ayoola OO, Salako AA, Idowu BM. Ultrasound evaluation of obstructive uropathy and its hemodynamic responses in southwest Nigeria. Int Braz J Urol. 2015; 41:556-61.

5. Platt JF, Rubin JM, Ellis JH. Distinction between obstructive and nonobstructive pyelocaliectasis with duplex Doppler sonography. AJR Am J Roentgenol. 1989; 153:997-1000.

6. Cubuk A, Yanaral F, Ozgor F, et al. Comparison of 4.8 Fr and 6 Fr ureteral stents on stent related symptoms following ureterorenoscopy:
A prospective randomized controlled trial. Kaohsiung J Med Sci. 2018; 34:695-9.

7. Tanidir Y, Mangir N, Sahan A, Sulukaya M. Turkish version of the Ureteral Stent Symptoms Questionnaire: linguistic and psychometric validation. World journal of urology. 2017; 35:1149-54.

8. Song T, Liao B, Zheng S, Wei Q. Meta-analysis of postoperatively stenting or not in patients underwent ureteroscopic lithotripsy. Urol Res. 2012; 40:67-77.

9. Haleblian G, Kijvikai K, de la Rosette J, Preminger G. Ureteral stenting and urinary stone management: a systematic review. J Urol. 2008; 179:424-30.

10. Piazzese EM, Mazzeo GI, Galipo S, et al. The renal resistive index as a predictor of acute hydronephrosis in patients with renal colic. J Ultrasound. 2012; 15:239-46.

11. Norris CS, Pfeiffer JS, Rittgers SE, Barnes RW. Noninvasive evaluation of renal artery stenosis and renovascular resistance. Experimental and clinical studies. J Vasc Surg. 1984; 1:192-201.

12. Patti G, Menghini ML, Todini AR, et al. The role of the renal resistive index ratio in diagnosing obstruction and in the follow-up of children with unilateral hydronephrosis. BJU Int. 2000; 85:308-12.

13. Tanidir Y, Sahan A, Asutay MK, et al. Differentiation of ureteral stones and phleboliths using Hounsfield units on computerized tomography: a new method without observer bias. Urolithiasis. 2017; 45:323-8.

14. Turk C, Petrik A, Sarica K, et al. EAU Guidelines on Diagnosis and Conservative Management of Urolithiasis. Eur Urol. 2016; 69:468-74.

15. Kilic S, Altinok MT, Ipek D, et al. Color Doppler sonography examination of partially obstructed kidneys associated with ureteropelvic junction stone before and after percutaneous nephrolithotripsy: preliminary report. Int J Urol. 2005; 12:429-35.

16. Klahr S, Pukerson ML. The pathophysiology of obstructive nephropathy: the role of vasoactive compounds in the hemodynamic and structural abnormalities of the obstructed kidney. Am J Kidney Dis. 1994; 23:219-23.

17. Seklehner S, Sievert KD, Lee R, et al. A cost analysis of stenting in uncomplicated semirigid ureteroscopic stone removal. Int Urol Nephrol. 2017; 49:753-61.

18. Ogreden E, Oguz U, Demirelli E, et al. The impact of ureteral Double-J stent insertion following ureterorenoscopy in patients with ureteral stones accompanied by perirenal fat stranding. Arch Ital Urol Androl. 2018; 90:15-19.

\author{
Correspondence \\ Bilal Eryildirim, $M D$ \\ Ahmet Sahan, MD \\ Özlem Türkoğlu, MD \\ Murat Tuncer, MD \\ Berkan Simsek, MD \\ Alkan Cubuk, MD (Corresponding Author) \\ alkancubuk@hotmail.com \\ Kartal Dr. Lutfi Kirdar Training a nd Research Hospital, Department of Urology \\ Cevizli Mh S.emsi Denizer Cad. E-5 Karayolu Cevizli Mevkii, \\ 34890 Kartal Istanbul (Turkey) \\ Övünç Kavukoğłu, MD \\ Health Sciences University, Kartal Dr. Lutfi Kirdar Training \\ and Research Hospital, Urology Clinic, Istanbul (Turkey) \\ Kemal Sarica, MD \\ Biruni University, Medical School, Urology Clinic, Istanbul (Turkey)
}

